\title{
Raising the suborbicularis oculi fat (SOOF): its role in chronic facial palsy
}

\author{
Jane M Olver
}

Western Eye Hospital, Marylebone Road, London NW1 5 YE and Eye Department, Charing Cross Hospital, Fulham Palace Road, London W6 8RF, UK J M Olver

Correspondence to: Marylebone Road, London NW1 5YE, UK

JaneOlver@aol.com

Accepted for publication 21 June 2000 Western Eye Hospital,

\begin{abstract}
Aims-To determine the adjuvant role of unilateral suborbicularis oculi fat (SOOF) lift in the periorbital rehabilitation of patients with chronic facial palsy.

Methods-In a non-comparative prospective case series nine adult patients (seven male, two female) aged 34-90 years (mean 60.5) with chronic unrecovered facial palsy (over 1 year), who had not had any previous rehabilitative periorbital surgery, were studied. Lateral tarsal strip and adjuvant transconjunctival approach subperiosteal SOOF lift under local or general anaesthesia were performed; medial canthoplasty was performed where indicated. There was clinical observation of the long term (over 1 year) effect on the ptotic palpebral-malar sulcus and lower eyelid retraction.
\end{abstract}

Results-The patients were followed up for 12-24 months (mean 16). Seven patients $(77 \%)$ had sustained clinical reduction of palpebral-malar sulcus ptosis. All patients had sustained reduction of lagophthalmos. Early postoperative complications included conjunctival cheimosis in $77 \%$. Three patients with persistent keratitis required further surgical procedures on their upper eyelid to reduce the palpebral aperture. There were no cases of infraorbital nerve anaesthesia or recurrent lower eyelid retraction.

Conclusions-The SOOF lift has an adjuvant role in chronic facial palsy with lower eyelid retraction and ptotic-palpebral malar sulcus. It supports the lower eyelid elevation and tightening achieved with the

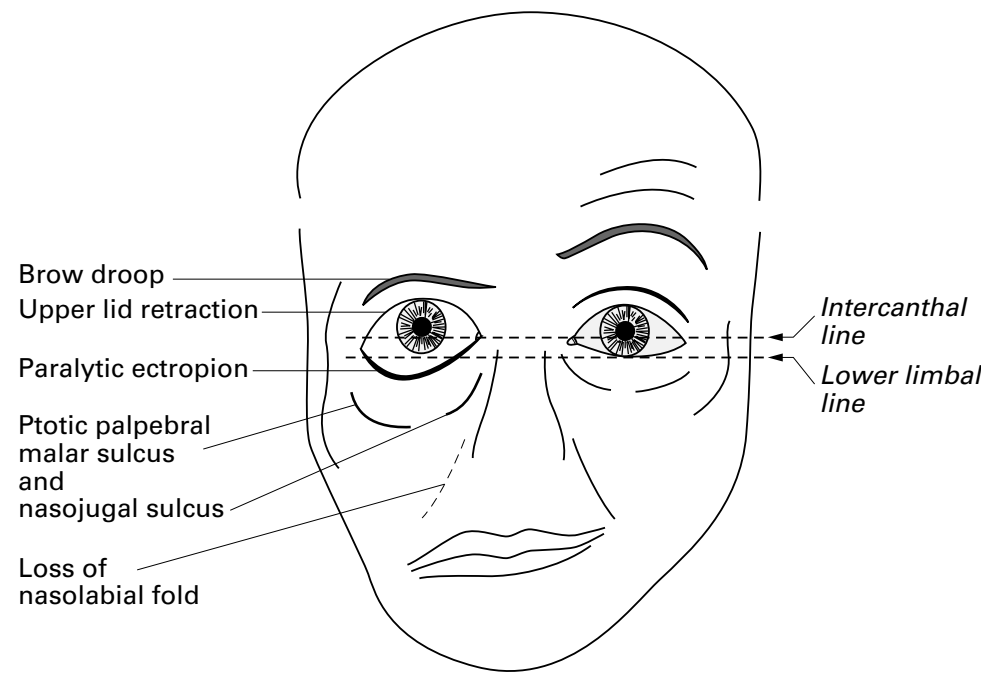

Figure 1 Diagram showing facial changes from lower motor neuron facial palsy. lateral tarsal strip. The best results were obtained in congenital facial palsy. (Br F Ophthalmol 2000;84:1401-1406)

The surgical management of chronic facial palsy is challenging and may involve several, staged operations by facial and oculoplastic surgeons. ${ }^{1-4}$ There is brow ptosis, lower eyelid retraction with horizontal eyelid laxity, lagophthalmos, prominent palpebral-malar and nasojugal sulci with hollowing of the infraorbital region where the lower eyelid and cheek meet, malar ptosis, and reduced nasolabial fold (Fig 1). Mild upper eyelid retraction and reduced blink rate and excursion also contribute to the lagophthalmos and potential keratitis. There may be associated reduction or absent cornea sensation resulting in neuropathic keratitis. The primary aim of oculoplastic rehabilitative surgery is to maintain good visual acuity by protecting the cornea by reducing lagophthalmos and the second aim, to produce a symmetrical periorbital and upper mid-face appearance. The lower eyelid is usually corrected before the upper eyelid and brow.

Lower eyelid surgery includes the lateral tarsal strip (LTS), possibly with retractor lysis or insertion of a hard palate mucosal graft. $^{5-7} \mathrm{~A}$ Lee medial canthoplasty reduces the medial vertical palpebral aperture when required. Other procedures such as lower eyelid fascial slings are reported to be effective. ${ }^{8}{ }^{9}$ These procedures do not address the ptotic palbebralmalar and nasojugal sulci or associated midface ptosis. Improving the position of the upper part of the cheek contributes to mid-facial symmetry as shown by pushing the cheek upwards and observing the effect on the lower eyelid and sulci between the cheek and the eyelid (Fig 2).

The layers of the cheek are skin, subcutaneous fat, orbicularis oculi and mimetic muscles. and suborbicularis fat. The suborbicularis fat pad (SOOF) lies in the suborbicularis, supraperiosteal plane, at and just below the inferior orbital rim on the zygoma and partly on the maxilla (Fig 3). The term SOOF was coined by aesthetic plastic surgeons in 1995, who would excise it at the time of blepharoplasty to avoid a contour defect. ${ }^{10}$

Repositioning (raising) the SOOF helps to elevate the overlying tissues, which in turn, helps to elevate the lower eyelid tissues, because they are a continuum. ${ }^{11}$ A LTS and adjuvant SOOF lift results in a myocutaneous redrape superiorly and superolaterally, since the anterior and posterior lamellae of the cheek are raised simultaneously. It is used by plastic and oculoplastic surgeons in the correction of 

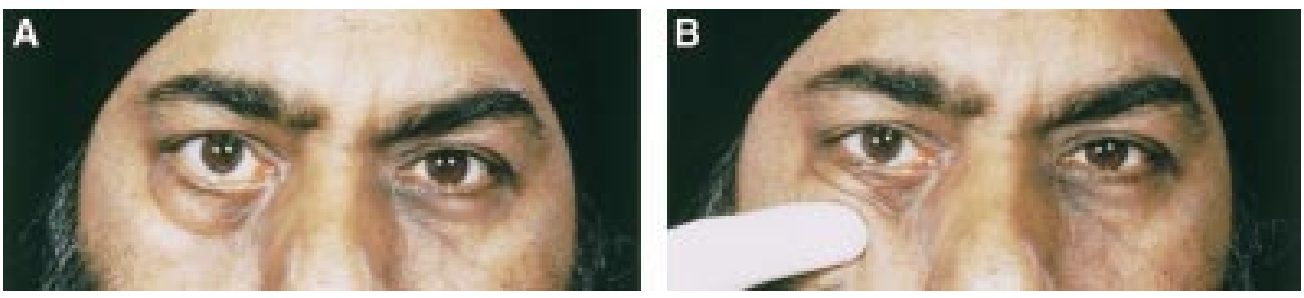

Figure 2 (A) Right congenital facial palsy. (B) Effect of digitally pushing the upper lateral cheek superior, mimicking the predicted effect of a SOOF lift.

cicatricial lower eyelid ectropion including post-blepharoplasty, correction of ageing midface ptosis, ${ }^{11}$ and providing additional tissue for reconstruction following tumour excision and loss of tissue from trauma.

The aim of this small study was to describe the effect of raising the suborbicularis oculi fat on the functional and cosmetic rehabilitation of patients with chronic facial palsy. In particular, it aimed to identify complications and determine whether the apparent beneficial effect on the lower eyelid and the palpebralmalar and nasojugal sulci was sustained. The ideal surgical outcome following an adjuvant SOOF lift would be to (a) regain symmetry of the lower eyelid position, palpebral-malar and nasojugal sulci and upper mid-face, (b) reduce lagophthalmos and provide good corneal protection, (c) have a rapid postoperative recovery with a low complication rate, and (d) be effective over a period of time.

\section{Methods}

The study design was a prospective noncomparative case series. Consecutive patients with long standing facial palsy (over 1 year) who had not had any previous eyelid surgery were included. Only those patients where pushing the cheek superior had a transmitted

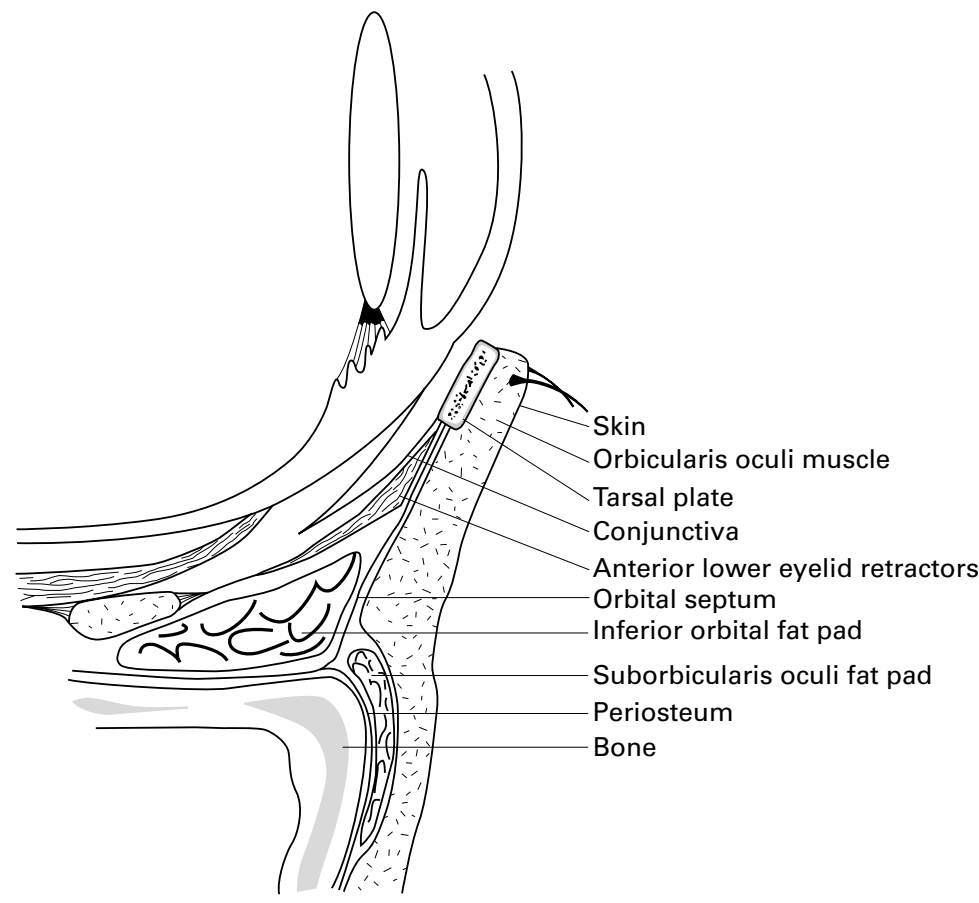

Figure 3 Cross section of a normal lower eyelid showing the location of the suborbicularis oculi fat (SOOF). effect of elevating the lower eyelid were deemed suitable-that is, the mid-face and lower eyelid acted as a continuum.

Preoperative assessment included evaluating facial asymmetry, measuring the palpebral aperture and lagophthalmos in $\mathrm{mm}$, evaluating the corneal exposure, detecting corneal anaesthesia, and determining the Bell's phenomenon.

All surgery was performed by an oculoplastic trained ophthalmologist (JMO).

The surgery consists of a lateral canthal approach via a $10-12 \mathrm{~mm}$ skin-orbicularis incision. After lateral canthotomy and cantholysis the lower part of the lateral orbital rim and the inferior orbital rim is approached via a subtarsal transconjunctival incision into the preseptal plane ${ }^{1213}$ (Fig 4A). The free lower conjunctival edge and retractors are placed on a 7.0 absorbable suture, draped superior over the cornea during the procedure. The periosteum is incised just anterior to the inferior orbital rim and elevated off the maxillary and zygomatic bones. The subperiosteal dissection is up to the lower border of the maxilla and zygoma (virtually to the gingival fornix, from where the dissector should be palpable). The lower edge of the periosteum is freed to elevate the cheek. The subperiosteal dissection preserves the infraorbital nerve, and only passes medial to the nerve where there is a noticeable ptotic nasojugal sulcus (Fig 4B). Between one and six non-absorbable sutures were placed in the periosteum and SOOF about $1 \mathrm{~cm}$ below the orbital rim and advanced superiorly and superolaterally to the arcus marginalis and to the superficial temporal fascia. The orbital rim was not drilled in this study. The conjunctiva is closed with absorbable 7.0 suture. A standard lateral tarsal strip (LTS) is made, titrating the length of the strip to the amount of horizontal eyelid laxity, with a small skin/muscle and eyelash margin excision laterally. Additional procedures include Lee medial canthoplasty and medial canthal tendon plication in selected cases.

The postoperative management protocol included eye padding for 24-48 hours, chloromycetin drops and dexamethasone drops four timed daily for 2-3 weeks. Follow up was at 1 , 2 , and 4 weeks, then at approximately 3,6 , and 12 months postoperatively. Longer follow up was available in some patients.

\section{Results}

Nine patients with chronic unrecovered facial palsy were included. The duration of the palsy exceeded 1 year in all patients. There were 


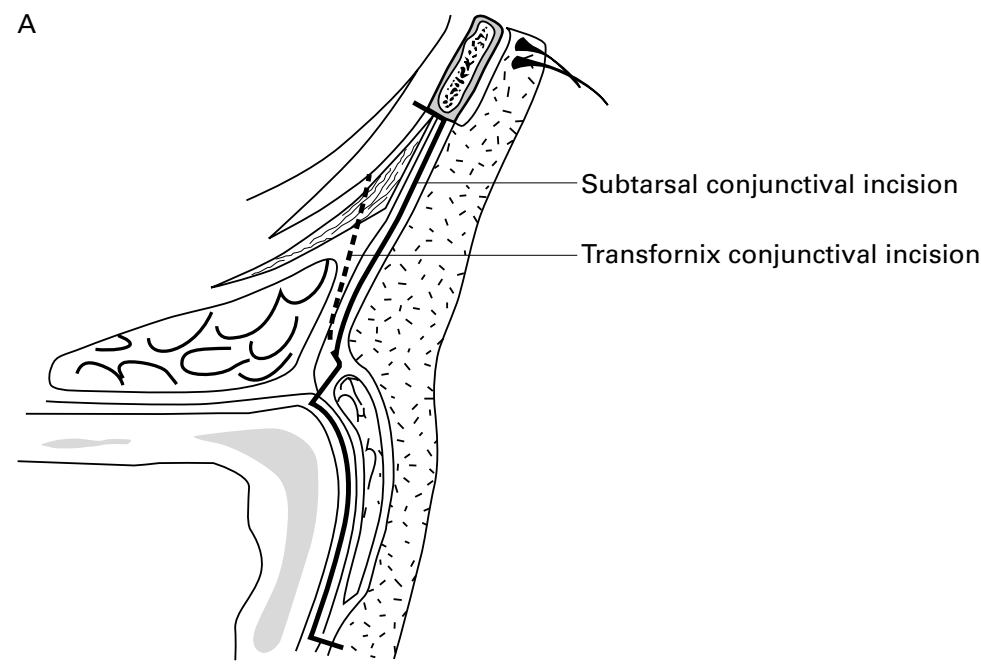

B

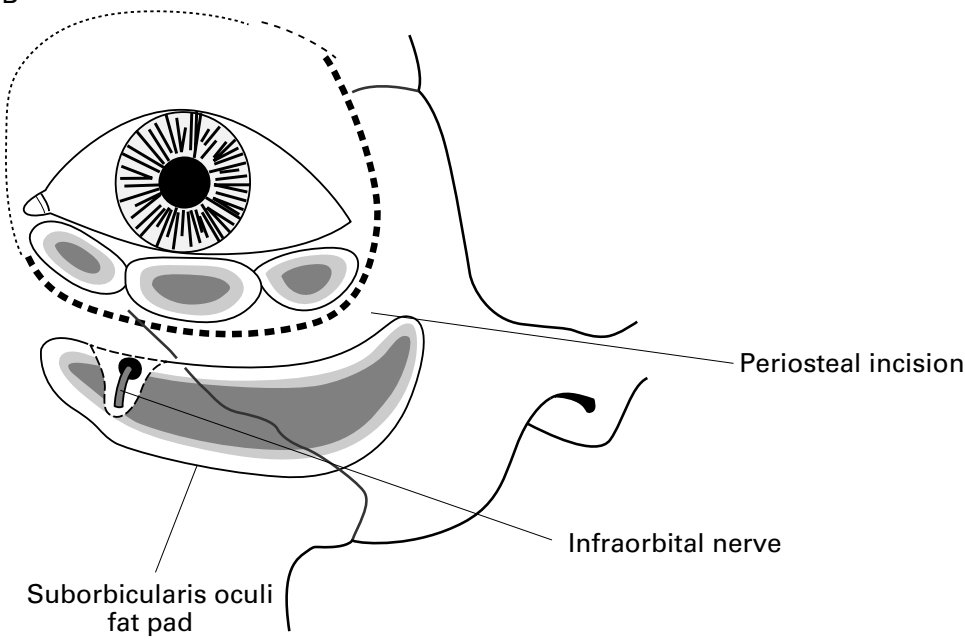

Figure 4 (A) Diagram showing transconjunctival approach to the inferior orbital rim and suborbicularis oculi fat (SOOF). The dissection continues posterior to the SOOF in the subperiosteal plane. (B) Diagram of left orbit showing location of SOOF in relation to the infraorbital nerve. The SOOF usually lies just above the infraorbital rim, but is shown

lower here, mimicking involutional or paralytic changes.

seven males and two females, aged $34-90$ years (mean 60.5). Follow up ranged from 12 to 24 months (mean 16). The aetiology of the facial palsy was congenital (four patients), posttumour (four patients), and unrecovered Bell's palsy (one patient). Five patients had 5.0 polyester sutures and four had 5.0 polypropylene sutures to suspend the SOOF. Between one and six sutures were used per operation (mean 3.5).

Surgery was performed under local anaesthesia, without additional sedation, in five patients and general anaesthesia in four patients.

The preoperative findings, operations performed, before, and 6 month postoperative lagophthalmos and the postoperative complications are summarised (Fig 5). Examples of three preoperative and postoperative appearances are shown (Figs 6, 7, and 8).

Most patients $(7 / 9,77 \%)$ had conjunctival chemosis in the first 2 weeks after surgery. One patient $(11 \%)$ had an early postoperative lateral canthal suture infection 1 week after surgery. There was persistent keratitis requiring subsequent eyelid procedures in three patients (33\%), two of whom had absent corneal sensation and one, a poor Bell's phenomenon. There was recurrent malar ptosis with prominent palpebral-malar sulci in two patients, both within 6 months of surgery - one requested a repeat procedure. In the remaining patients there was little apparent further palpebral-malar sulcus drop between 6 and 12 months. Lagophthalmos was stable by 6 months, unless further surgery was done on the upper eyelid-for example, insertion of a gold weight, which further reduced the palpebral aperture. The patients with congenital facial ptosis had the best, sustained malar improvement with reduction of hollowing and sag in the infraorbital region where the lower eyelid and cheek meet. There was no loss of sensation in the distribution of the infraorbital nerve or recurrent lower eyelid retraction.

\section{Discussion}

The suborbicularis oculi fat (SOOF) is a periorbital fat pad similar to the retro-orbicularis oculi fat (ROOF) pad in the brow. ${ }^{14}{ }^{15}$ Anatomists regard it as a deeper part of the malar fat pad which is mainly anterior to the mimetic muscles zygomaticus major and levator labii superioris. ${ }^{15} 16$ With age, there is a gradual descent of the orbicularis and, with it, the SOOF. ${ }^{13}$ This gives the typical appearance of an involutional mid-face ptosis with a prominent palpebral malar fold, where there is sagging cheek skin and hollowing in the infraorbital region between the eyelid and cheek. With involutional mid-face ptosis there is exaggeration of the nasolabial fold, while in facial palsy, this is softened and elongated. In chronic facial palsy, there is a paralytic mid-face ptosis, prominent palpebral-malar sulcus, facial asymmetry, and lower eyelid retraction and lagophthalmos; descent of the paralytic orbicularis occurs, with or without SOOF descent. The mid-facial fat compartments (malar and SOOF), are normally bound to the orbicularis muscle by the superficial aponeurotic system. ${ }^{15}$ Therefore, if the paralytic orbicularis has dropped, the SOOF is likely to have descended with it unless there is slippage between the lamellae secondary to orbitomalar ligament weakening. Redraping the SOOF superiorly is usually accompanied by redraping of the overlying orbicularis and skin, thereby moving inferior periorbital skin upwards into the lower eyelid. ${ }^{13}$ The lateral tarsal strip corrects the horizontal eyelid laxity and is supported vertically by the myocutaneous redrape from the SOOF lift.

Correction of paralytic lower eyelid retraction by a lateral tarsal strip alone is effective in tightening the lower eyelid horizontally, and is a simple quick procedure which can easily be done under local anaesthesia. The main drawback of the LTS used alone is that it does not always elevate the lower eyelid adequately, nor correct the associated palpebral-malar sulcus defect. Since the LTS has no effect on the 

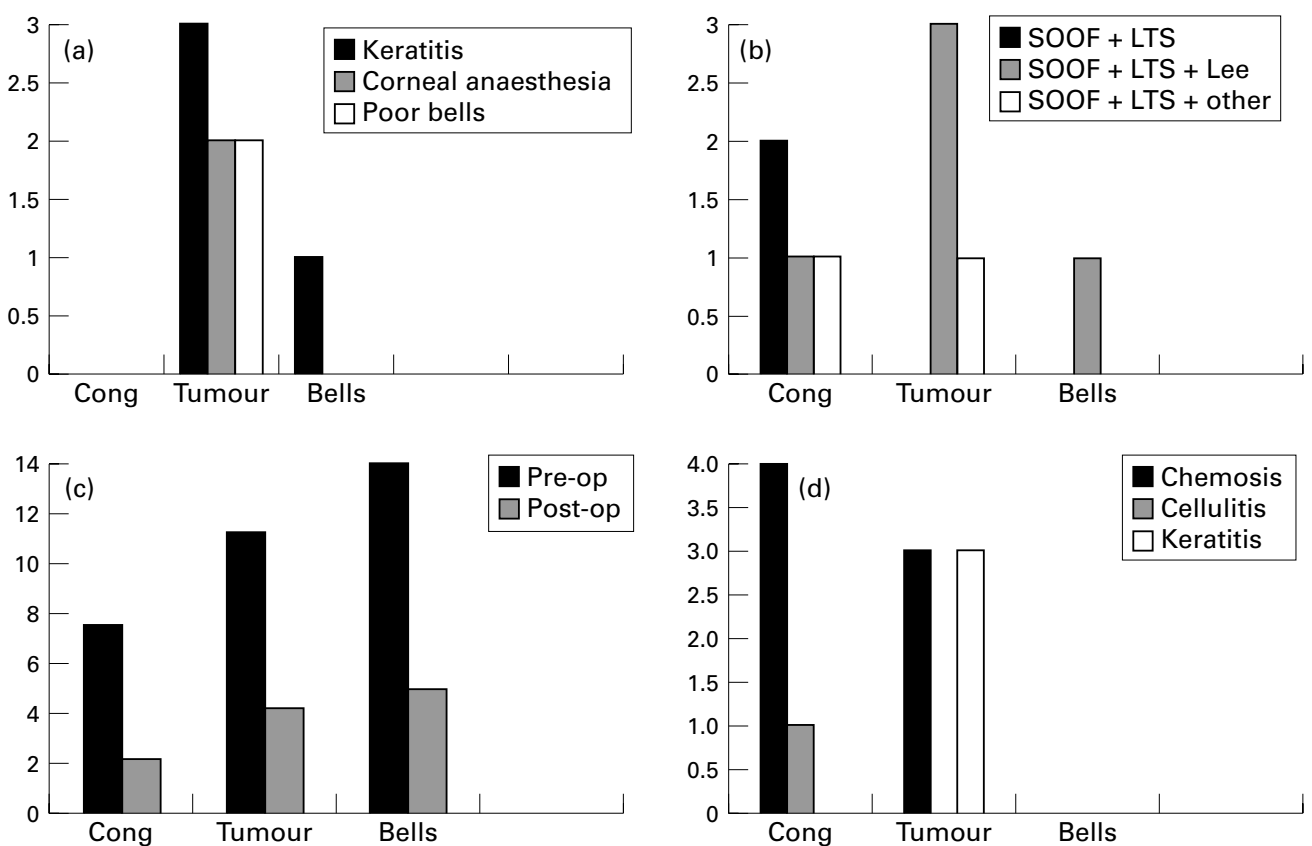

Figure 5 (A) Preoperative clinical findings. (B) Combination of procedures performed. SOOF $=$ suborbicularis oculi fat, LTS = lateral tarsal strip, Lee $=$ Lee medial canthoplasty. Other is orbital fat excision (1) and medial canthal tendon stabilisation for medial canthal tendon laxity (1). (C) Measurement of preoperative lagophthalmos and residual lagophthalmos 6 months after surgery, in mm. (D) Early postoperative complications.

mid-face ptosis, the cosmetic facial improvement is not as good as with an adjuvant SOOF lift.

In this study, subperiosteal dissection was predominantly prezygomatic and malar, lateral to the infraorbital nerve, to elevate the palpebral-malar sulcus and support the lower eyelid elevation, hence there were no cases of postoperative mid-facial anaesthesia. More extensive subperiosteal dissection medial to the
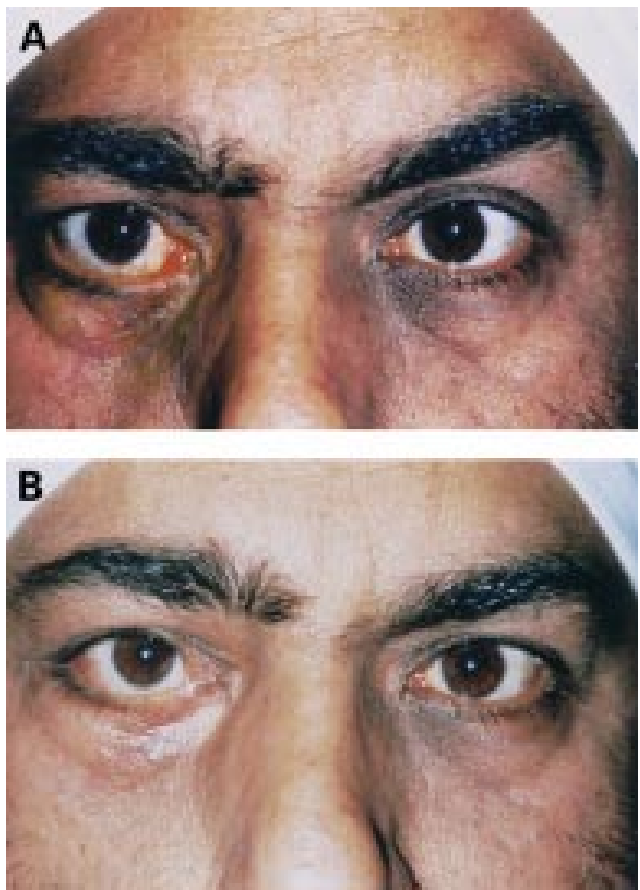

Figure 6 (A) Preoperative appearance of right congenital facial palsy with prominent palpebral-malar ptosis. (B)

Same patient, 1 year after left LTS, Lee medial canthoplasty, and SOOF lift, showing sustained improvement in palpebral-malar symmetry. infraorbital nerve, right down to the level of the buccal mucosa above the incisor was not attempted, particularly as many of the patients had their surgery done under local anaesthesia. More extensive dissection may partially improve lower mid-face ptosis, but carry a greater risk of infraorbital nerve damage. This study shows that, for the best results, a minimum of three elevating sutures are required, more if the tissue is heavy.
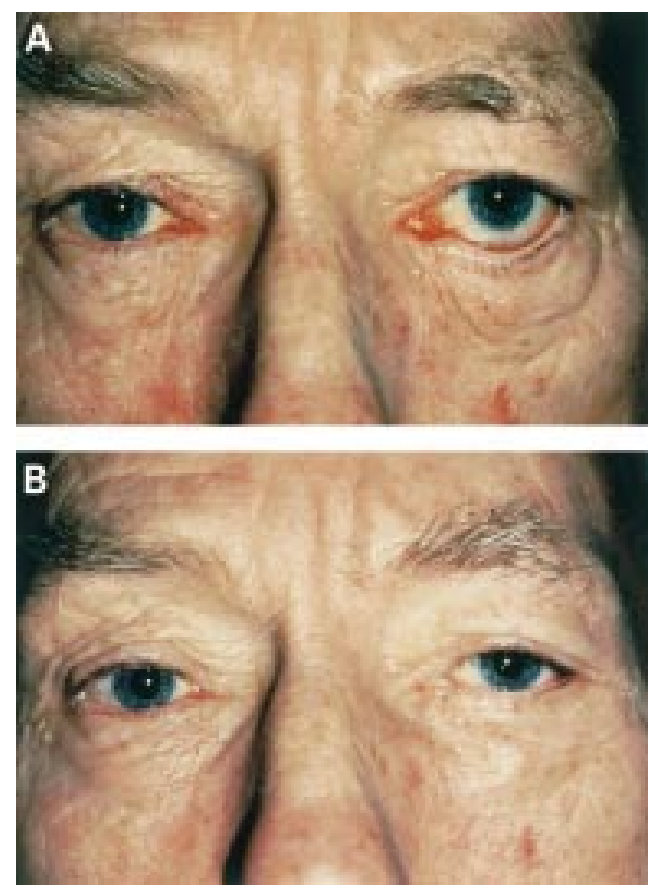

Figure 7 (A) Preoperative appearance of left acquired facial palsy with lower eyelid retraction and palpebral malar ptosis. (B) Same patient, showing a good result 1 year after left LTS and SOOF lift. 

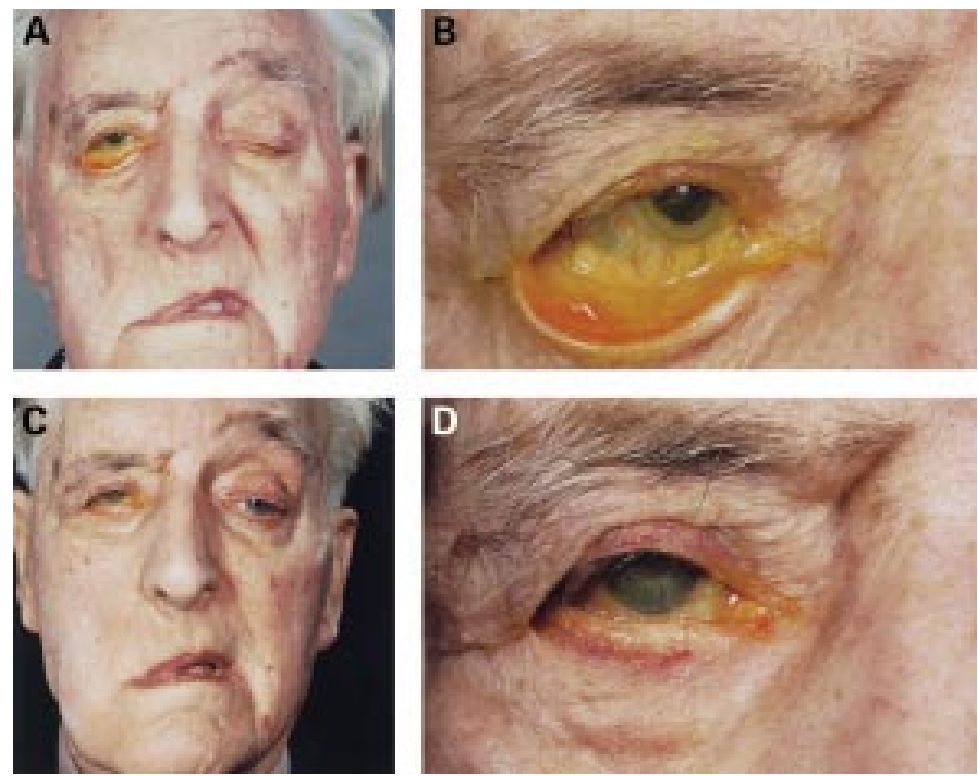

Figure 8 ( $A, B)$ Preoperative appearance of right long standing Bell's palsy with marked lower eyelid retraction and ptotic palpebral-malar ptosis. $(C, D)$ One month postoperative appearance with improved lower eyelid position and elevated upper cheek after right LTS and SOOF lift. There was a neuropathic cornea and this patient required later upper eyelid lowering for corneal protection.

Correction of the lower eyelid retraction and mid-face ptosis in facial palsy is challenging and may involve a combination of several procedures to achieve both adequate corneal protection and a satisfactory aesthetic result. Ideally, the number of operations should be kept to a minimum and be effective with a low morbidity. In this study, LTS with adjuvant SOOF lift was beneficial in the rehabilitation of $7 / 9$ patients with chronic facial palsy. The best effect was in congenital cases where the cheek tissue is thinner and atrophic, therefore easily redrapes. If the tissue is heavy, subsequent drop is greater.

Although the SOOF lift supports elevation of the lower eyelid, it does not prevent exposure keratitis in severe acquired cases, where additional procedures are required, even at the expense of a good cosmetic appearance and symmetry. If there is reduced corneal sensation, the vertical palpebral aperture must be left narrower on that side for corneal protection. In this study, the patients with congenital facial palsy had no keratitis preoperatively, normal corneal sensation and good Bell's phenomenon, and symmetry was achieved.

It is extremely difficult to measure the success of a SOOF lift objectively. Results are based on observations by the surgeon, aided by photographic documentation, and the opinion of the patient. Both surgeon and patient agreed there was anatomical success in 7/9 patients over 1 year after surgery. Two patients had recurrent malar ptosis - one had only had one suture placed in the SOOF, which proved inadequate, the other had a very heavy cheek and a greater lift should have been done. Although the number of patients in this study was small with only medium term follow up, the series suggests that adjuvant SOOF lift is effective. A longer follow up would help to determine whether the effect is sustained after several years, as the natural course would be for some further palpebral-malar sulcus drop with time, and that more patients would need repeat surgery. It would also be valuable to compare the LTS alone with LTS and adjuvant SOOF lift.

The commonest complication of the SOOF lift in this series was postoperative chemosis in the early postoperative period. Transconjunctival access to the inferior and inferolateral orbital rim has been previously reported to cause conjunctival chemosis, ${ }^{17}$ presumably secondary to the interruption of lower eyelid lymphatic drainage. One patient had a temporary suture abscess. The postoperative protocol has changed following this study, to include flurbiprofen $50 \mathrm{mg}$ three times daily starting on the fifth day after surgery for 5-7 days, and a broad spectrum antibiotic for 5 days after surgery, to address the chemosis and risk of infection.

Surgical techniques for raising the SOOF are evolving. Aesthetic surgeons have described raising the SOOF superolaterally to the deep temporal fascia just lateral to the lateral orbital rim, using an endoscopic technique via a 1.5 $\mathrm{cm}$ crow's foot incision or an upper buccal mucosal incision. ${ }^{14-19}$ The concavity over the inferior orbital rim can also be corrected by repositioning the orbital extraconal fat anteriorly over the rim. ${ }^{20-23}$ Another technique used in blepharoplasty, of suturing the capsulopalpebral fascia to the arcus marginalis after limited excision of inferior extraconal fat, may also be beneficial if there is prominent orbital fat prolapse accentuating the malar ptosis. ${ }^{24} 25$

The transconjunctival SOOF lift with a standard LTS, has a role in improving the periorbital function and cosmesis in the rehabilitation of patients with chronic facial palsy, particularly those with congenital facial palsy. Oculoplastic surgeons can apply the surgical techniques used by the aesthetic facial plastic surgeons to improve the lower eyelid retraction and palpebral-malar ptosis in facial palsy.

This paper was presented, in part, at the European Society of Ophthalmic Plastic and Reconstructive Surgery in Rome, October 1997

1 Adenis JP, Mathon C. Traitement chirurgical de la lagophtalmie. $\mathcal{F}$ Fr Ophtalmol 1988;11:91-103.

2 Leatherbarrow B, Collin JRO. Eyelid surgery in facial palsy. Eye 1991;5:585-90.

3 Seiff S, Chang J. Management of ophthalmic complications of facial nerve palsy. Otolaryngol Clin N Am 1992;25:66990 .

4 Sadiq SA, Downes RN. A clinical algorithm for the management of facial palsy from an oculoplastic perspective. Eye 1998;2:219-23.

5 Holds JB, Anderson RL, Thiese SM. Lower eyelid retraction: a minimal incision surgical approach to retraction: a minimal incision surgical appro

6 Bartley GB, Kay PP. Posterior lamellar eyelid reconstruction with a hard palate mucosal graft. Am $\mathcal{f}$ Ophthalmol 989;107:609-12.

7 Shorr N. Madame Butterfly procedure with hard palate graft: management of postblepharoplasty round eye and scleral show. Facial Plast Surg 1994;10:90-118.

8 Wiggs EO, Guibor P, Hecht SD, et al. Surgical treatment of the denervated or sagging lower lid. Ophthalmol 1982;89: 428-32.

9 Gilliland GD, Wobig JL, Dailey RA. A modified surgical technique in the treatment of facial nerve palsies. Ophthalmic Plast Reconstr Surg 1998;14:94-8.

10 Aiache AE, Ramirez OH. The suborbicularis oculi fat pads. Plast Recontr Surg 1995;95:37-42.

11 Hoenig JA, Shorr N, Shorr J. The suborbicularis oculi fat in aesthetic and reconstructive surgery. Int Ophthalmol Clin 1997;37:179-91. 
12 McCord CD, Moses JL. Exposure of the inferior orbit with fornix incision and lateral canthotomy. Ophthalmic Surg fornix incision

13 McCord CD, Codner MA, Hester TR. Redraping the inferior orbicularis arc. Plast Reconstr Surg 1998;107:2471-9.

14 Ramirez OM. The subperiosteal approach for the correction of the deep nasolabial fold and the central third of the face. Clin Plastic Surg 1995;22:341-56.

15 Kikkawa DO, Lemke BN, Dortzbach RK. Relations of the superficial musculoaponeurotic system to the orbit and characterisation of the orbitomalar ligament. Ophthalmic Plast Reconstr Surg 1996;12:77-88.

16 Owsley JQ. Lifting the malar fat pad for correction of prominent nasolabial folds. Plast Reconstr Surg 1993;91: 463-74.

17 Westfall CT, Shore JW, Nunery WR, et al. Operative complications of the transconjunctival inferior fornix approach. cations of the transconjunctival
Ophthalmology 1991;98:1525-8.

18 Fuente del Campo A Subperiosteal facelift: open and endoscopic approach. Aesth Plast Surg 1995;19:149-60.
19 Ramirez OM, Pozner JN. Sub-periosteal minimally invasive laser endoscopic rhytidectomy: the SMILE facelift. Aesth Plast Surg 1996;20:463-70.

20 Loeb R. Fat pad sliding and fat grafting for leveling lid depressions. Clin Plast Surg 1981;8:757-8.

21 Hamra ST. Repositioning the orbicularis oculi muscle in the composite rhytidectomy. Plast Reconstr Surg 1992;90:1422.

22 Hamra ST Arcus marginalis release and orbital fat preservation in midface rejuvenation. Plast Reconstr Surg 1995;96:354-62.

23 Hamra ST. The role of orbital fat preservation in facial aesthetic surgery. Clin Plastic Surg 1996;23:17-28.

24 De la Plaza R, Aroyo JM. A new technique for the treatment of palpebral bags. Plast Reconstr Surg 1988;81:677.

25 Camirand A, Doucet J, Harris J. Anatomy, pathophysiology, and prevention of senile enophthalmia and associated herniated lower eyelid fat pads. Plast Reconstr Surg 1997;100: 1535-46. 